\title{
Paragangliomas of the Spine
}

\author{
Guohua LV' ${ }^{1 *}$, Lingyun $\mathrm{LU}^{2^{*}}$, Zhehao DAI ${ }^{1}$ \\ ${ }^{1}$ The Second Xiangya Hospital of Central South University, Department of Spine Surgery, Changsha, China \\ ${ }^{2}$ The Fifth Hospital of Xiamen, Department of Orthopedics, Xiamen, China \\ *Guohua LV and Lingyun LU contributed equally to this work.
}

\section{ABSTRACT}

AIM: Paragangliomas of the spine are rare tumors. Clinical presentations and courses of spinal paragangliomas are varied, and there are no standard principles of treatment to date. The purpose of this study was to explore the diagnosis, treatment and prognosis of spinal paragangliomas.

MATERIAL and METHODS: The clinical data of 7 consecutive cases, with complete medical records and follow-up results that were treated in our institutions from October 2000 to October 2011, was retrospectively reviewed.

RESULTS: There were 6 males and 1 female with a mean age of 40 years (range, 16-61). The follow-up period ranged from 40 to 98 months (mean, 72 months). Of the 6 primary spinal paragangliomas, one lesion was in the cervical intradural extramedullary space, one in the thoracic epidural space and four in the lumbar intradural extramedullary space. All tumors were totally resected and no recurrence was detected during the follow-up period. Of the metastatic case, the lesion of the spine was located in the first lumbar epidural space and vertebra. The patients underwent surgical resection two times with radiotherapy, but the tumor recurred and the patient suffered from the paraplegia of lower limbs and urine and stool incontinence during the follow-up period. No patient died.

CONCLUSION: Spinal paragangliomas are rare lesions and seldom considered in the presurgical differential diagnosis due to its rarity and non-specific clinical symptoms and imaging features. Clinical follow-up was necessary to determine the outcome. Complete resection is necessary to prevent recurrence. The role of radiotherapy in the management of these lesions needs further assessment.

KEYWORDS: Paraganglioma, Spine, Metastasis

\section{INTRODUCTION}

$\mathrm{P}$ aragangliomas are rare tumors that arise from the paraganglia cells of the neuroendocrine system and account for $0.3 \%$ of all neoplasms $(2,4)$. More than $80 \%$ of this kind of tumor develops within or near the glomus jugulare and carotid body. Paragangliomas of spinal involvement are extremely rare. Primary spinal paragangliomas usually take the form of intradural compression of the cauda equina or intra/extradural compression of the thoracic and cervical spinal cord (1). The first case of metastatic spread of a paraganglioma to the spine was reported in 1948. So far, less than 30 cases of spinal metastases by paragangliomas have been reported in English.
Clinical presentations and courses of spinal paragangliomas are varied, and there are no standard principles of treatment to date $(1,8)$. In this article, we report the clinical data of 7 consecutive cases with complete medical records and followup results that were treated in our institutions, in an attempt to explore the diagnosis, treatment and prognosis.

\section{MATERIAL and METHODS}

A retrospective analysis was performed in 7 patients with spinal paraganglioma in our hospital from October 2000 to October 2011. No patient was lost in the follow-up period. There were 6 cases of primary spinal paraganglioma and one case 
of metastatic spread of a paraganglioma to the lumbar and thoracic spine from the retroperitoneum (Figure 1A, B). The demographic data, clinical presentation, radiological presentation, distribution, treatment methods and outcome, and histopathology analysis were reviewed.

\section{RESULTS}

\section{Clinical Presentation}

The age of the patients ranged from 16 to 61 (mean: 40 years), with a male predominance $(M / F=6 / 1)$. The presentation of these spinal paragangliomas varied by location of the tumor, but local pain with a gradual and insidious onset was the most common symptom, which occurred in all patients, and was followed by limitation of spinal activity. The duration of pain before a definitive diagnosis is established varied from 2 weeks to 10 years (mean: 35.6 months). In the metastatic case, a $10 \mathrm{~cm} \times 8 \mathrm{~cm}$ stiff, non-tender, smooth and fixed mass at the left hypochondriac region and a $2 \mathrm{~cm} \times 2 \mathrm{~cm}$ mass at the first lumbar (L1) vertebra level were palpated. Neurological symptoms were seen in all cases (Table I). No adrenergic symptoms were found in any case.

\section{Radiographic Presentation}

Plain radiographs showed no abnormality in 5 cases, while there was the sign of bone destruction in L1 in the metastatic case and L4 lumbar spondylolisthesis in the remaining case. Furthermore, lateral radiography of the upper cervical and upper thoracic areas was usually of poor quality. In the metastatic case, computed tomography (CT) showed a 105 $\mathrm{mm} \times 67 \mathrm{~mm} \times 80 \mathrm{~mm}$ well circumscribed mass with soft tissue density, which compressed the tail of the pancreas, the spleen, aorta and left kidney (Figure 2A, K). The mass was heterogeneous, and had a solid parenchyma with some cystic changes. Spotted calcification could be seen. The tumor was irregularly enhanced after contrast injection. The posterior edge of the L1 vertebra, both vertebral arches, the spinous process, left transverse process, and articular process had spotted osteonecrosis (Figure 2A, K). The CT scans found no abnormality in the affected region in the remaining 6 cases. The magnetic resonance imaging (MRI) scans of the lesions were characterized by iso- or hypointense signals on T1-weighted images and high- or isointense signals on T2-weighted images that enhanced with intravenous administration of gadolinium. All the mass except the metastatic one had an irregular oblong oval-shape in the vertebral canal and compressed the spinal cord, cauda equina or nerve root. A paravertebral soft tissue mass were seen in the metastatic case only.

\section{Distribution}

There were 7 lesions distributed along the spinal column in 7 patients. Of the 6 primary spinal paragangliomas, one lesion was found in the cervical intradural extramedullary space, one in the thoracic epidural space (Figure 1A, B) and four in the lumbar intradural extramedullary space (Figure 3A, B). Of the metastatic case, the lesion of the spine was located in the first lumbar epidural space and vertebra (including vertebral body and posterior elements) simultaneously at the time of diagnosis.

Table I: Clinical Data on 7 Patients with Spinal Paragangliomas

\begin{tabular}{|c|c|c|c|c|c|c|c|}
\hline Case & Sex & $\begin{array}{c}\text { Age } \\
\text { (years) }\end{array}$ & History & Location & Treatment & Evolution & $\begin{array}{l}\text { Follow-up } \\
\text { (years) }\end{array}$ \\
\hline 1 & M & 16 & $\begin{array}{c}\text { Hypaesthesia of the trunk below } \\
\text { cartilago ensiformis (xiphoid process) } \\
\text { and numbness and weakness of the } \\
\text { both legs for } 2 \text { weeks }\end{array}$ & T6-7, extradural & Total resection & DFS & 8.2 \\
\hline 2 & $\mathrm{~F}$ & 38 & $\begin{array}{l}\text { Lumbago on the left for } 5 \text { months, } \\
\text { numbness and weakness of the both } \\
\text { legs for } 19 \text { days }\end{array}$ & $\begin{array}{l}\mathrm{L} 1 \text {, metastatic } \\
\text { spread from } \\
\text { retroperitoneum }\end{array}$ & $\begin{array}{l}\text { Subtotal } \\
\text { resection and } \\
\text { radiotherapy }\end{array}$ & $\begin{array}{l}\text { Local recurrence+ } \\
\text { progression }\end{array}$ & 8 \\
\hline 3 & $M$ & 61 & $\begin{array}{l}\text { Lumbago and numbness of the both } \\
\text { legs for } 5 \text { years }\end{array}$ & L2-3, intradural & Total resection & DFS & 7.2 \\
\hline 4 & M & 52 & $\begin{array}{l}\text { Lumbago and posterior thigh pain for } \\
\qquad 10 \text { years }\end{array}$ & L2-3, intradural & Total resection & DFS & 7 \\
\hline 5 & $M$ & 40 & $\begin{array}{l}\text { Lumbar pain and left leg pain for } 2 \\
\text { months. }\end{array}$ & L2, intradural & Total resection & DFS & 4.2 \\
\hline 6 & $M$ & 37 & $\begin{array}{l}\text { Increasing neck pain over a period of } \\
2 \text { years, numbness and unendurable } \\
\text { pain of the right maxillofacial region } \\
\text { for } 1 \text { month. }\end{array}$ & C2-3, intradural & Total resection & DFS & 4 \\
\hline 7 & M & 36 & $\begin{array}{c}\text { Lumbago and both legs pain for } 3 \\
\text { years. }\end{array}$ & L2-3, intradural & Surgery & DFS & 3.3 \\
\hline
\end{tabular}

F: Female, M: Male, DFS: Disease-free survival. 


\section{Treatment}

All patients underwent surgical intervention. A posterior approach was employed for tumor resection in the primary cases, and spinal stability was reconstructed by internal fixation. For the metastatic one, the retroperitoneal tumor was resected though the explorative laparotomy first. Two weeks later, L1 laminectomy was performed; the posterior elements of L1, which were visibly infiltrated with tumor and the solid tumor adhesion to the left L1 nerve root, were resected. As fragments of tumor that extended to the anterior portion of the L1 vertebra were not excised, percutaneous vertebroplasty (PVP) with polymethyl methacrylate (PMMA) was done under $\mathrm{C}$-arm $\mathrm{X}$-ray guidance. Then, reconstruction with transpedicular screw instrumentation was performed. ${ }^{131}$ I MIBG therapy $(11.1 \mathrm{GBq}$ ) and external beam radiotherapy (40 Gy in 15 fractions) were applied postoperatively.

\section{Biopsy}

All patients had a pathologic diagnosis of paraganglioma during surgery.

\section{Clinical Follow-up Period}

The 7 patients were followed up for 40 months to 98 months (mean, 72 months). The results showed good outcomes in

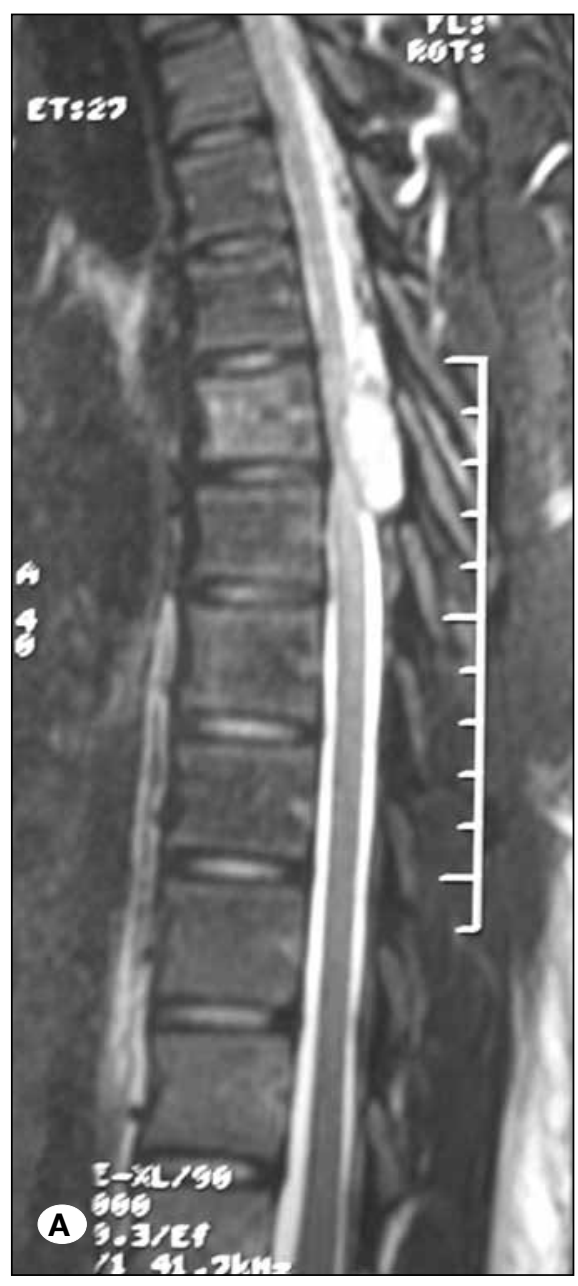

the primary cases in which the symptoms disappeared and who had no evidence of active disease at the latest clinical follow-up. Poor outcomes were seen in the metastatic patient. Fifty months after the first surgery, a new lesion was found in the left appendix vertebra of T10-11 with extension into the spinal canal and paravertebral soft tissue, and she underwent surgical resection of the lesion and external beam radiotherapy to at L1 and T10 at our institutions. Six months later, a local recurrence in the left T10 and T11 intervertebral foramen with extension to the vertebral canal was seen. Further surgery was refused. The patient is still alive and no more metastatic site could be found elsewhere at the last follow-up, but she suffered from the paraplegia of lower limbs and urine and stool incontinence.

\section{DISCUSSION}

Paragangliomas are rare tumors that arise from the paraganglia cells of the neuroendocrine system. Although paragangliomas may occur in any age group, they are mainly seen in adults, and are uncommon in children and adolescents. In our study, only one patient was under 18 years of age and the male/ female ratio was $6: 1$. More than $80 \%$ of paragangliomas, generally corresponding to the designation chemodectomas, occur in or near the glomus jugulare and carotid body. Spinal paragangliomas are extremely rare. To date, approximately 250 cases have been reported and the vast majority were intradural in the cauda equina. In our study, only one lesion was found in the cervical intradural extramedullary space and one in the thoracic epidural space. Some prior case reports indicated that thoracic paragangliomas are as a group distinct from the paragangliomas of the cauda equina in that they are generally extradural and have a greater predilection for distant metastasis (3).

The best-known type of paraganglioma is the pheochromocytoma, which secretes catecholamines and leads to labile hypertension. Although all paraganglia sequester catecholamines in cytoplasmic granules, paragangliomas arising from non-adrenal tissue are rarely active in neuroendocrine production (10). Only a few cases of spinal paraganglioma have been

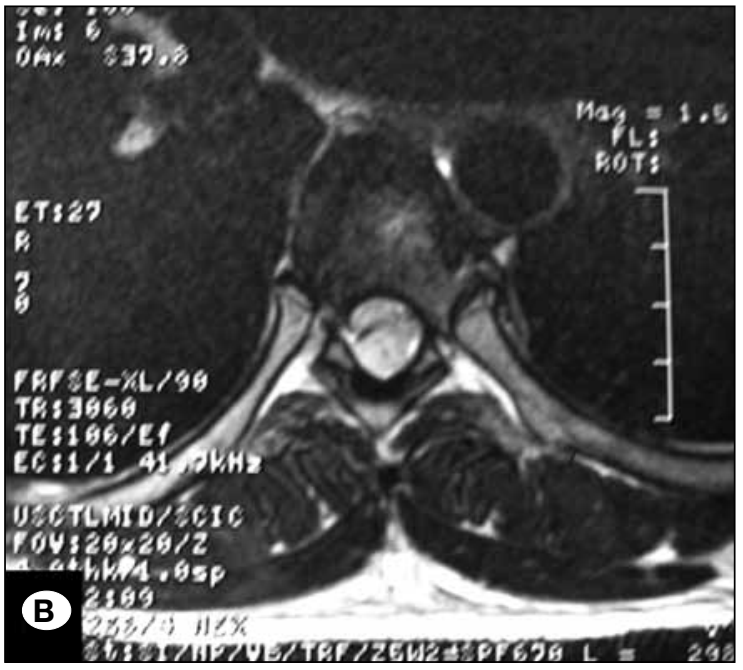

Figure 1: (Case 1) Magnetic Resonance Imaging A) T2weighted sagittal image, showing a well circumscribed, homogeneously enhancing extradural tumor filling the spinal canal at the T6-7 level. B) Axial T2-weighted image, showing the mass lesion displaced the cord to the front of the spinal canal. 

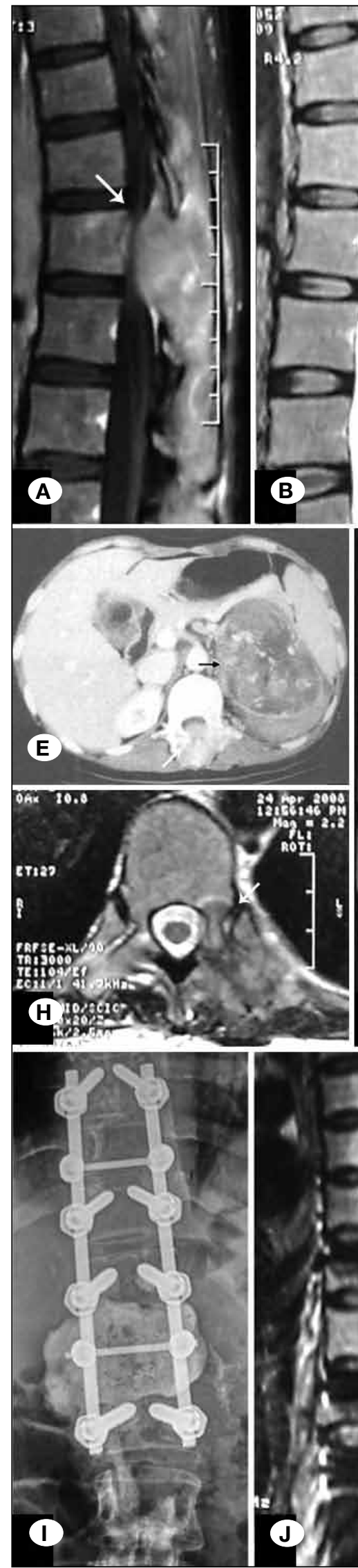
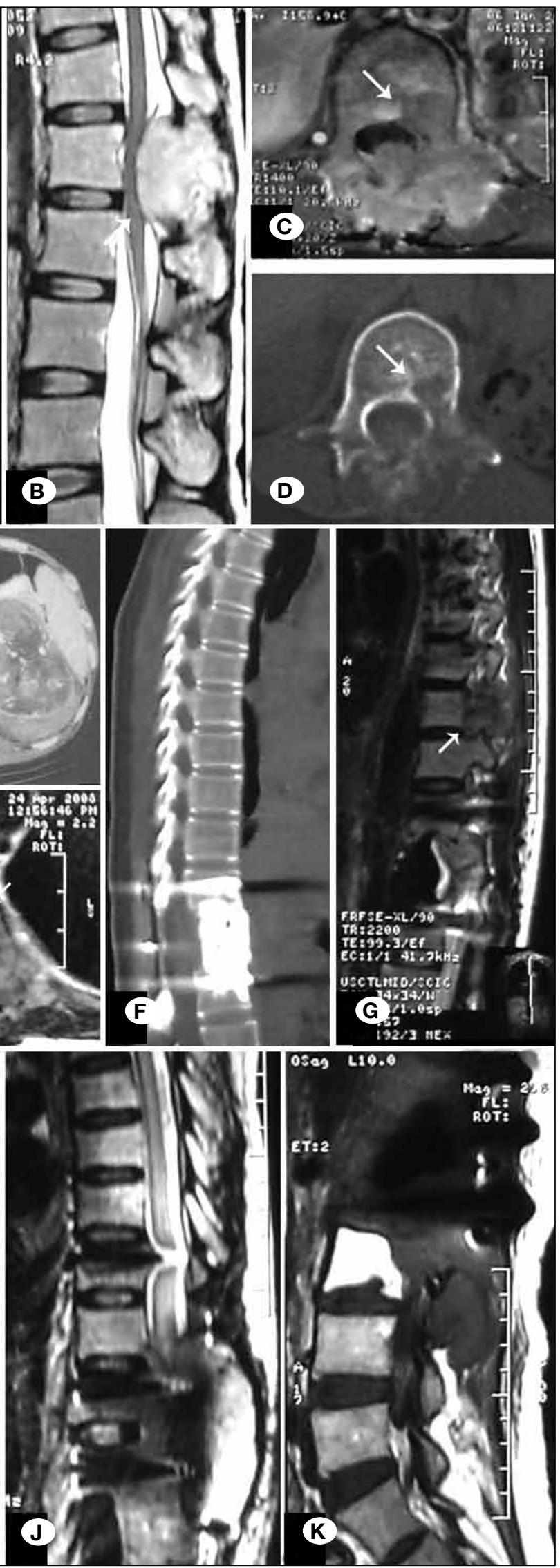

Figure 2: (Case 2) A) T1-weighted sagittal image and B) T2-weighted sagittal image showing spinal cord extradural compression at the vertebral body of $\mathrm{L} 1$ (white arrow). C) Axial magnetic resonance image and D) CT showing the posterior edge of L1 vertebrae, both vertebral arches, spinous process, left transverse process, and articular process had spotted osteonecrosis (white arrow). E) CT scan of abdomen showing a well-circumscribed, heterogeneous mass that was irregularly enhanced at 80.05 Hounsfield units after contrast injection, and compressed the tail of pancreas, spleen, aorta and left kidney (black arrow). The posterior edge of L1 vertebrae, both vertebral arches, spinous process, left transverse process, and articular process had spotted osteonecrosis (white arrow). F) CT scanning after the first surgery, showing no loosening and rupture of the screws, and no local recurrence. G) Sagittal MRI 50 months after the first surgery, showing a new mass of appendix of $\mathrm{T} 10-11$, and $\mathbf{H}$ ) axial MRI revealing an infiltrative tumor in the left appendix vertebra of T10-11 with extension into the spinal canal and to the paravertebral soft tissue. I) Anterior-posterior radiograph demonstrating no loosening and rupture of the screws after the second surgery. J, K) MRI 6 months after the second surgery revealing that the spinal canal between T10 and L2 was filled with tumor, extending through the foramina to the paravertebral soft tissue. 
reported to secrete catecholamines $(1,2,16)$. In our study, all the cases were non-functional paragangliomas, and vanillylmandelic acid and catecholamine were in normal range in the urine and blood of these patients. For those patients with a non-functional paraganglioma, it was difficult to make a correct preoperative diagnosis due to the absence of typical clinical symptoms. Local dull pain was the most common symptom, and other clinical manifestations included limited motion, radiating pain, and limb paresis, which was due to spinal cord or nerve root compression. The neurological dysfunctions were the main reasons why patients went to hospital for medical help.
The origin of primary paraganglioma in the spine is uncertain, since the existence of paraganglia cells in the CNS remains unclear. Sundgren et al. (17) suggest that a spinal paraganglioma may originate from sympathetic neurons in the thoracic and lumbar lateral horns of the spinal cord or heterotopic neurons, which lie along these branches proximal to the sympathetic trunk. Guevara et al. (7) believed that paraganglioma developed from the incomplete regression of fetal paraganglia, with persistence of vestigial tissue till adult life.

MRI examination is sensitive in detecting the mass and could delineate its location, outline, internal structure as well as

Table II: Immunohistochemical Features of 7 Cases

\begin{tabular}{ccccccccccc}
\hline Case & S100 & Vimentin & NSE & Cytokeratin & EMA & Chromogranin Synaptophysin & Nf & CD34 & CD68 & Ki67 \\
\hline $\mathbf{1}$ & \pm & + & ++ & - & - & & - & - \\
\hline $\mathbf{2}$ & + & +++ & - & - & - & + & + & - & - \\
\hline $\mathbf{3}$ & - & + & + & - & - & + & + & - & - \\
\hline $\mathbf{4}$ & + & & + & + & + & + & + & \\
\hline $\mathbf{5}$ & + & & + & Focal+ & - & + & + & \\
\hline $\mathbf{6}$ & ++ & & & - & & + & + & + \\
\hline 7 & ++ & ++ & ++ & - & Focal+ & ++ & ++ & + \\
\hline
\end{tabular}

NSE: Neuron-specific enolase, Nf: Neurofilament proteins, EMA: Epithelial membrane antigen.
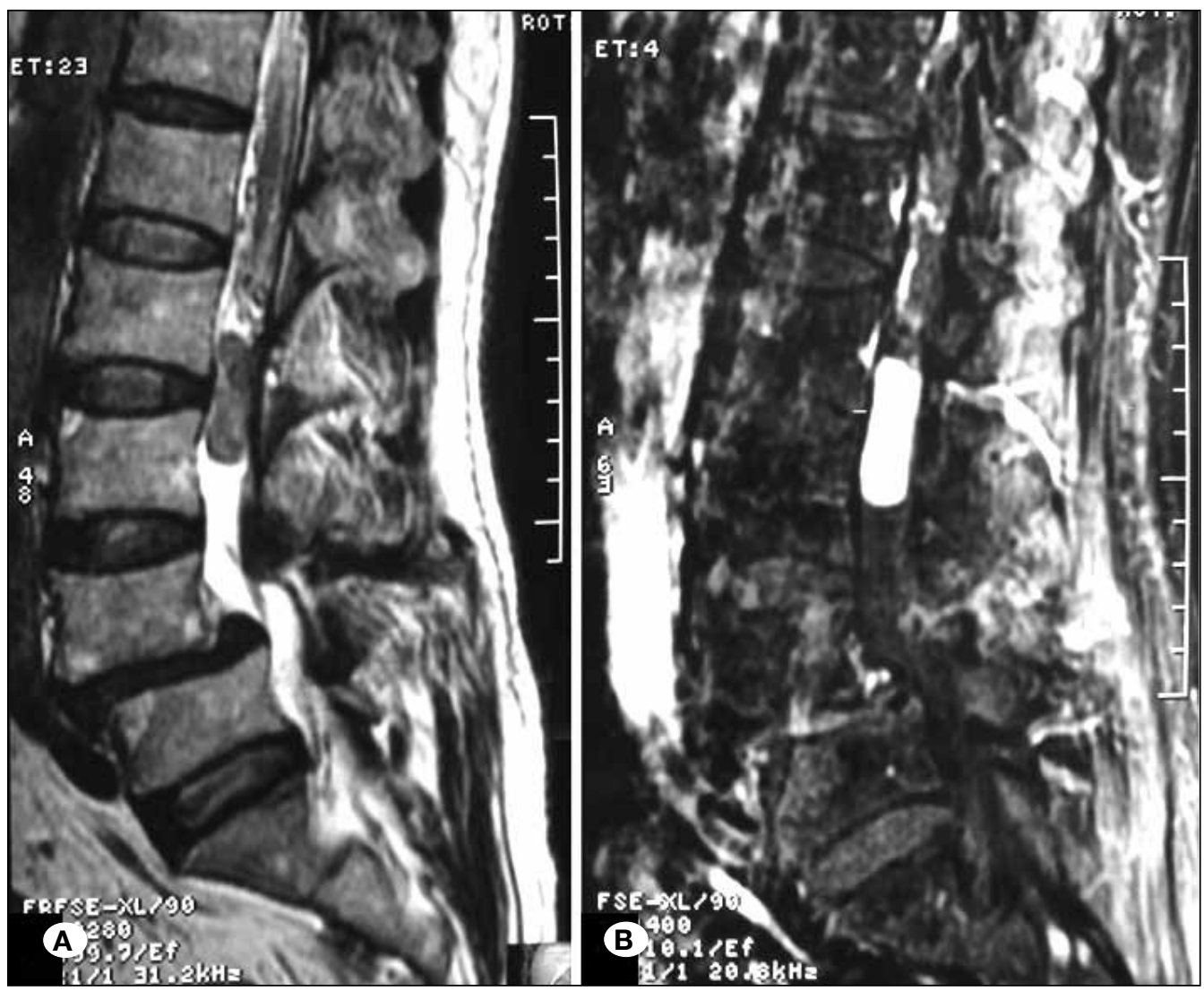

Figure 3: (Case 4) Magnetic Resonance Imaging

A) Sagittal T1-Weighted image, showing a wellcircumscribed, intradural tumor isointense to the spinal cord in the spinal canal at the L2/L3 level.

B) Sagittal T1-Weighted image with gadolinium showing that the mass is strongly homogeneously enhanced. 
its relationship with the surrounding tissue and organs. T1weighted images give better spatial resolution and better show the highly vascular internal matrix of the tumor. T2weighted images give better tissue contrast, making smaller lesions more conspicuous. In general, the tumors exhibited relatively prolonged $\mathrm{T} 1$ and $\mathrm{T} 2$ relaxation times. On T1weighted images, 3 tumors were of approximately equal signal intensity to adjacent muscle, while 4 tumors were slightly higher in intensity than surrounding muscle. On T2weighted images, 5 tumors showed variable but greater signal intensity than muscle, while 2 tumors were of equal signal intensity. All the masses enhanced with intravenous administration of gadolinium. The T1-weighted and T2weighted imaging characteristics differentiated dermoid tumor and lipoma from paragangliomas. In 4 of the 7 tumors, there were multiple punctate and serpiginous areas of signal void, named "salt-and-pepper" appearance, due to highvelocity flow in tumor vessels seen on T2-weighted imaging. Because of the hypervascular nature, the "salt-and-pepper" appearance on T2-weighted imaging was characteristic of paragangliomas. However, this appearance can also be seen in other hypervascular tumors such as hemangioblastoma, angioma, and metastatic thyroid carcinoma, which are more common in the spine region.

The diagnosis of paraganglioma still relies on the postoperative histological examination. Paragangliomas have a variety of histological growth patterns, but the characteristic "Zellballen" pattern, a nesting or clustering of cell groups, and the trabecular cords of cells within thin compartments of connective tissue stroma, is the primary histological feature of paragangliomas (14). In our study, 4 of the 7 tumors presented with the above characteristic morphologic appearance. The ependymoma, the main lesion in the differential diagnosis, is characterized by perivascular pseudorosettes, radially oriented cell groups surrounding small vessels. When none of the above classical patterns is found, it is very difficult to differentiate paragangliomas from ependymomas on routine staining. Immunohistochemical staining was most commonly used for differential diagnosis. Chromogranin, which is demonstrated in granules, and synaptophysin, a membrane glycoprotein specific for presynaptic vesicles (5), and neuron specific enolase, are positive in paragangliomas, whereas negativity for glial fibrillary acidic protein exclude ependymomas with ease. Table II summarizes the result of the immunohistochemical staining. It is difficult to differentiate between benign and malignant tumors, though several reviews have examined series of paraganglioma to determine gross features, microscopic characteristics and biologic actions that might help predicting which ones have a propensity for metastatic spread. Both tumors show cellular pleomorphism and local or vascular invasion (4). Clinical follow-up was necessary to determine the outcome.

Total removal of the tumor was adequate treatment and the goal of surgery. The recurrence for the metastatic patient was due to incomplete removal. When removal of the entire tumor is not possible, radiation treatment is recommended $(9,18)$. The general recommended dose is 4,000 to 4,500 cGy. However this is largely based on studies done on giant cell tumors and chordomas $(6,9)$. The use of chemotherapy is controversial. U-King-Im et al. (18) reported successful therapeutic use of octreotide in a patient with carotid body paraganglioma that metastasized to multiple vertebrae. Mertens demonstrated the successful regression and palliation of metastases of paraganglioma with chemotherapy, particularly with combined cyclophosphamide, vincristine and dacarbazine (13). However, there were also a number of disappointing reports on chemotherapy for this type of tumor $(8,11,12,15)$. In our study, 6 patients with primary paraganglioma only underwent surgery and the follow-up showed good outcomes and no recurrence. For our metastatic case, postoperative radiotherapy seemed to be of no value in preventing local recurrence. Subtotal resection was the main reason for the tumor recurrence. Consecutive radiotherapy should be restricted to malignant or recurring paragangliomas.

\section{- CONCLUSION}

Spinal paragangliomas are very rare lesions. They will seldom be considered in the pre-surgical differential diagnosis due to their rarity and non-specific clinical symptoms and imaging features. The diagnosis is made by histopathological examination. Clinical follow-up is necessary to determine the outcome. Complete resection is necessary to prevent recurrence. The role of radiotherapy in the management of these lesions needs further assessment and should be restricted to malignant or recurring paragangliomas.

\section{ACKNOWLEDGEMENT}

This research was approved by the ethics committee of Central South University. The manuscript does not contain any information about medical devices/drugs. No funds and benefits were received in support of this work. None of the authors have any possible conflicts of interest.

\section{REFERENCES}

1. Boker DK, Wasseman H, Solymosi L: Paragangliomas of the spinal canal. Surg Neurol 19: 461-468, 1983

2. Chen G, Luo Z, Liu T, Yang H: Functioning paraganglioma of the cervical spine. Orthopedics 34:e700-702, 2011

3. Conti P, Mouchaty H, Spacca B, Buccoliero AM, Conti R: Thoracic extradural paragangliomas: $A$ case report and review of the literature. Spinal Cord 44:120-125, 2006

4. Duke WW, Boshell BR, Soteres P: A norepinephrine secreting glomus jugulare tumor presenting as a paraganglioma. Ann Intern Med 60: 1040-1047, 1964

5. Fitzgerald LF, Cech DA, Goodman JC: Paraganglioma of the thoracic spinal cord. Clin Neurol Neurosurg 98:183-185, 1996

6. Gibbs IC, Chang SD: Radiosurgery and radiotherapy for sacral tumors. Neurosurg Focus 15(2):E8, 2003

7. Guevara N, Chays A, Bruzzo M, Meller R, Balabasard CF, Magnan J: Cerebellopontine angle paraganglioma. Otol Neurotol 24:469-472, 2002

8. Hamilton MA, Tait D: Metastatic paraganglioma causing spinal cord compression. Br J Radiol 73:901-904, 2000 
9. Jindel R, Gupta AK, Mahapatra AK, Bal CS, Singhal RM: Extradural paraganglioma with multiple skeletal metastases. Br J Radiol 65:938-940, 1992

10. Kleiwer KE, Cochran AJ: A review of the histology, ultrastructure, immunohistochemistry and molecular biology of extra-adrenal paragangliomas. Arch Pathol Lab Med 113: 1209-1218, 1989

11. Konowitz PM, Lawson W, Som PM, Urken ML, Breakstone BA, Biller HF: Laryngeal paraganglioma: Update on diagnosis and treatment. Laryngoscope 98:40-49, 1988

12. Majumdar S, Friedrich CA, Koch CA, Megason GC, Fratkin JD, Moll GW: Compound heterozygous mutation with a novel splice donor region DNA sequence variant in the succinate dehydrogenase subunit $B$ gene in malignant paraganglioma. Pediatr Blood Cancer 54:473-475, 2010

13. Mertens WC, Grignon DJ, Romano W: Malignant paraganglioma with skeletal metastases and spinal cord compression: Response and palliation with chemotherapy. Clin Oncol 5:126-128, 1993
14. Moran CA, Rush W, Mena H: Primary spinal paragangliomas: A clinicopathological and immunohistochemical study of 30 cases. Histopathology 31:167-173, 1997

15. Richter A, Halm HF, Lerner T, Liljenqvist UR, Quante M: Longterm follow-up after en bloc resection and reconstruction of a solitary paraganglioma metastasis in the first lumbar vertebral body: A case report. J Med Case Rep 5:45, 2011

16. Simpson LN, Hughes BD, Karikari IO, Mehta Al, Hodges TR, Cummings TJ, Bagley CA: Catecholamine-secreting paraganglioma of the thoracic spinal column: Report of an unusual case and review of the literature. Neurosurgery 70:E1049-1052, 2012

17. Sundgren P, Annertz M, Englund E, Strömblad LG, Holtås S: Paragangliomas of the spinal canal. Neuroradiology 41:788794, 1999

18. U-King-Im JM, Carroll TA, Morris K: Vertebral metastatic chemodectoma: Imaging and therapeutic octreotide. Case report. J Neurosurg Spine 97:106-109, 2002 